\title{
El papel del branding en el marketing de las organizaciones culturales
}

\author{
Teresa López-Doriga Casanueva \\ Birkbeck College, University of London
}

\begin{abstract}
RESUMEN: Art Basel y ARCOmadrid son los dos casos de estudio utilizados en este artículo para analizar cómo una marca fuerte basada en un buen producto, es vital para el desarrollo de una estrategia de marketing exitosa en un segmento del panorama cultural tan íntimamente ligado al mundo de los negocios como son las ferias de arte.
\end{abstract}

PALABRAS CLAVE: Feria de Arte, Marketing, Art Basel, ARCOmadrid, Coleccionismo, Arte Contemporáneo.

\section{Branding Strategies in Arts Marketing}

\begin{abstract}
Art Basel and ARCOmadrid are the two case studies employed in this article to show how a strong brand based on a good product is essential for developing a successful marketing strategy. Moreover, this is particularly relevant in a cultural segment linked to the business world as art fairs are.
\end{abstract}

KEY WORDS: Art Fair, Marketing, Art Basel, ARCOmadrid, Art Collection, Contemporary Art.

Recibido: 3 de abril de 2013 / Aceptado: 30 de junio de 2014.

Branding y marca, en el más amplio sentido del término, deben tener un papel determinante en el marketing de cualquier organización relacionada con el arte. En un sector tan vasto como el cultural, una marca fuerte basada en un buen producto es esencial para el desarrollo de una estrategia de marketing exitosa. Estas marcas culturales deben ser consistentes y hablar de los valores únicos de la compañía, posicionándola como la primera opción en la mente del consumidor. A lo largo de este artículo, la comparación de dos ferias de arte, ARCOmadrid y Art Basel, servirá de base para tres puntos clave: abordar la siempre difícil cuestión de la definición de marca; demostrar la importancia fundamental del branding en una estrategia de marketing exitosa; y analizar cómo y por qué el branding se usa en un segmento del panorama cultural tan íntimamente ligado al mundo de los negocios como son las ferias de arte.

\footnotetext{
* LÓPEZ-DORIGA CASANUEVA, Teresa: «El papel del branding en el marketing de las organizaciones culturales", Boletín de Arte, n. ${ }^{\circ}$ 35, Departamento de Historia del Arte, Universidad de Málaga, 2014, pp. 169-186, ISSN: 0211-8483.
} 


\section{Definición de marca}

Cuando se habla de marca, es una palabra extraña, porque es bastante impreciso lo que la gente quiere decir con ella Matthew Slotover, copropietario de Frieze

La definición de "marca", sobre todo cuando se hace referencia a una marca cultural, es un reto. No hay una respuesta clara o simple, porque, entre otras cosas, estamos hablando de un concepto complejo que ha evolucionado de forma extraordinaria.

Como Hyland \& King señalan, "hasta hace poco, la palabra "marca" se refería simplemente a un símbolo que identificaba un producto o un fabricante» ${ }^{1}$. Sin embargo, actualmente es una definición bastante limitada ya que sólo hace referencia a la parte tangible. Además de los elementos visuales, una marca "es el mayor activo de muchas organizaciones de arte ${ }^{2} y$ «una promesa que verbaliza quién eres, qué haces y porqué eso es importante», además de "una fuente de eficacia porque actúa como un dispositivo de ahorro de tiempo, proporcionando un atajo en el proceso de toma de decisiones de potenciales inversores, clientes y socios» ${ }^{3}$.

Por lo tanto, en el sector cultural, "una marca representa todo lo que un producto o servicio significa para el usuario» 4 . Una marca es un elemento muy complejo que traduce la misión y la visión de la organización en aspectos tangibles, y tiene que ser clara, precisa, consistente, única, emocional y estable. Además, al aplicar la marca al sector cultural hay que considerar la naturaleza particular del producto cultural y ser capaz de crear una imagen coherente basada en un producto dinámico e innovador que ofrece al consumidor una experiencia personal enriquecedora.

1 HYLAND, Angus y KING, Emily, Visual identity and branding for the arts (online), Laurence King, Londres, en: http://books.google.es/books?hl=es\&id=Q3NPAAAAMAAJ\&focus=searchwithinvolume\&q =trademark (22-junio-2014).

2 COLBERT, François y FOURIER, Marilyne, "An experimental investigation of the use of brand extension and co-branding strategies in the arts", Journal of Services Marketing (online), vol. 21, n. ${ }^{\circ} 4$, pp. 231-240, en: Proquest/ABI/INFORM Global (18-abril-2012).

3 KYLANDER, Nathalie y STONE, Chistopher, "The role of brand ing the non-profit sector", Stanford Social Innovation Review (online), en: http://bc2.berkeley.edu/wp-content/uploads/2012/07/the-role-ofbrand-in-the-nonprofit-sector.pdf (22-junio-2014).

4 ARMSTRONG, Gary y KOTLER, Philip, Principles of Marketing (online), Pearson, Londres, en: http:// books.google.es/books?id=ZW2u5LOmbs4C\&printsec=frontcover\&hl=es\#v=onepage \&q\&f=false $(22$-junio-2014). 


\section{Art Basel versus ARCOmadrid: dos marcas culturales}

La mayoría de las características ya mencionadas son de aplicación a las dos organizaciones culturales utilizadas como casos de estudio en este artículo: Art Basel y ARCOmadrid. Estas marcas, no son tan populares como Tate o MoMA, sino que su conocimiento implica una relación directa con el mundo de las artes plásticas.

Art Basel asegura en su página web que es «la muestra más importante a nivel mundial de arte internacional moderno y contemporáneo". La feria, que se celebra anualmente en junio, comenzó en 1970 como una alternativa internacional a Art Colonia (enfocada exclusivamente al arte alemán), y se mantuvo relativamente desconocida hasta 1991, cuando Lorenzo A. Rudolf asumió la dirección y la reinventó. Él fue el artífice de la rápida transformación de un encuentro puramente comercial a un amplio evento cultural, a base de cambios tan fundamentales como la designación de un comité de selección y del UBS como principal patrocinador. Desde 2007, los codirectores Annette Schönholzer y Marc Spiegler, han estado al frente de las seis secciones de la feria: Programa General, más de 300 galerías de 36 países; Art Statements, 26 propuestas individuales de artistas jóvenes; Art Features, 20 espacios centrados en la actividad de las galerías desde el punto de vista del comisario; Art Unlimited, 50 proyectos a gran escala, creados para la feria; Art Parcous, obras colocadas en la ciudad; y Eventos (Conversations, Art Salon, Art Film o Art on Stage). Además, 2002 fue el año de lanzamiento de Art Basel Miami, enfocado en el arte americano, y en 2011 se dio el siguiente paso con la expansión hacia el mercado asiático, adquiriendo Art Hong Kong. Otras ferias satélites como Scope Basel y Volta6 han aparecido también a lo largo de los últimos años.

ARCOmadrid es la feria de arte más visitada del mundo. Hasta 150.000 personas recorren sus pasillos durante sus 5 días de duración. Comenzó en 1982 y desde entonces ha tenido continuidad anual cada febrero, con éxito variable, y desde la edición de 2011, primera de Carlos Urroz como director, se divide en cinco secciones: Programa General (117 galerías de 21 países); País Invitado; Solo Proyects, centrado en propuestas de arte latinoamericano; Opening, para galerías de reciente creación; y Eventos (AfterARCO, ARCOkids o Experts Forum). El evento también ha generado dos ferias satélite: JUSTMAD desde 2009 y Art Madrid desde 2010.

Tanto Art Basel como ARCOmadrid tienen actualmente una clara identidad basada en la visión, valores y ambición de las organizaciones que las preceden y 
que son los pilares de su imagen de marca. En la mayoría de las organizaciones de arte esta parte intangible de la marca está definida y explicada en su página web, pero esto no sucede en ninguno de los dos casos a analizar. Sin embargo, se pueden deducir a partir de las webs de los sponsors, entrevistas con los directores (que son los máximos embajadores de la marca) y teniendo en cuenta los cambios que las ferias han ido llevando a cabo para adaptarse a los nuevos tiempos ${ }^{5}$ :

\begin{tabular}{|c|c|}
\hline VISIÓN & \\
\hline Art Basel & ARCOmadrid \\
\hline $\begin{array}{l}\text { Ser "la muestra más importante a nivel } \\
\text { mundial de arte internacional moderno } \\
\text { y contemporáneo", un evento en el que } \\
\text { todos los activos culturales de la zona } \\
\text { trabajan para conseguir una atmósfera } \\
\text { cultural gratificante para todos los } \\
\text { amantes del arte que visitan la histórica } \\
\text { ciudad de Basilea durante esos días. }\end{array}$ & $\begin{array}{l}\text { Ser «una de las mayores plataformas } \\
\text { del mercado del arte, al tiempo que un } \\
\text { evento imprescindible para la promoción } \\
\text { y difusión del arte emergente más allá de } \\
\text { la fronteras españolas y europeas». }\end{array}$ \\
\hline
\end{tabular}

\begin{tabular}{|l|l|}
\hline MISIÓN & \\
\hline Art Basel & ARCOmadrid \\
\hline "Continuar creando una experiencia \\
$\begin{array}{l}\text { artística global para los visitantes y no ser } \\
\text { sólo un espacio de compra-venta de arte». }\end{array}$ & $\begin{array}{l}\text { «Repensar todos los proyectos y } \\
\text { programas para crear una feria duradera, } \\
\text { sólida, contemporánea e internacional, } \\
\text { claramente enfocada a los ‘nuevos } \\
\text { coleccionistas'». }\end{array}$ \\
\hline
\end{tabular}

5 Para Art Basel la información se encuentra en: http://www.artbasel.com/; http://www.artinfo.com/ news/story/27747/annette-schnholzer-and-marc-spiegler/; http://www.artfacts.net/index.php/pageType/ newsInfo/newsID/4194/lang/1; http://www.artreview100.com/people/770/; http://www.artinamericamagazine.com/news-opinion/the-market/2009-06-10/a-conversation-with-marc-spiegler-co-director-of-artbasel/. Para ARCOmadrid la información se encuentra en: http://www.ifema.es/ferias/arco/default.html; http://www.guiadegalerias.com/en/contents/current-issues/item/51-entrevista-a-carlos-urroz-directorde-arco-2011. 


\begin{tabular}{|c|c|}
\hline VALORES & \\
\hline Art Basel & ARCOmadrid \\
\hline $\begin{array}{l}\text { Conector: el lugar donde yuxtaponer ideas } \\
\text { sobre arte con el arte en sí mismo. } \\
\text { Estabilidad: oferta de una sólida gama } \\
\text { de servicios y apoyo a las galerías } \\
\text { participantes (clave del éxito). } \\
\text { Innovación: } \\
\text { - liderazgo artístico: descubriendo nuevos } \\
\text { artistas. } \\
\text { - innovación tecnológica: ejemplarizada } \\
\text { en la aplicación interactiva del mapa 3D } \\
\text { que permite visitar la feria virtualmente, } \\
\text { ver y organizar piezas, y reenviar } \\
\text { información. } \\
\text { Excelencia: basada en un riguroso comité } \\
\text { de selección. } \\
\text { Panorámica de lo que ocurre en el arte a } \\
\text { nivel mundial. } \\
\text { Diversa: } \\
\text { - todas las formas de expresión artística, } \\
\text { creadas a partir de } 1900 \text {. } \\
\text { desde asequibles artistas emergentes a } \\
\text { obras maestras. }\end{array}$ & $\begin{array}{l}\text { Internacional: } \\
\text { - Presentaciones en el extranjero. } \\
\text { - Sección de País Invitado. } \\
\text { - América Latina: con Solo Project. } \\
\text { Vanguardista: promoviendo la creación } \\
\text { de arte a través, por ejemplo, del premio } \\
\text { ARCO/BEEP de arte electrónico. } \\
\text { Poliédrica: con facetas intelectuales } \\
\text { (Forum y Encuentros), sociales (Arco Kids) } \\
\text { y recreativas (AfterArco). } \\
\text { Diversa: con artistas establecidos y arte } \\
\text { emergente europeo y latinoamericano. } \\
\text { Moderna y revitalizada: nuevo diseño más } \\
\text { confortable para el visitante (pasando de } \\
\text { tres a dos pabellones). Refuerzo del uso de } \\
\text { comunicación digital introduciendo apps, } \\
\text { catálogo online y redes sociales. } \\
\text { Comprometida con galerías y } \\
\text { coleccionistas: desarrollo del programa } \\
\text { First Collectors y «del programa de } \\
\text { coleccionistas internacionales». }\end{array}$ \\
\hline
\end{tabular}

\begin{tabular}{|l|l|}
\hline METAS & \\
\hline Art Basel & ARCOmadrid \\
\hline - Mantener el nivel de calidad. & - Ser una plataforma que facilite las \\
- Expandir la marca Art Basel. & transacciones comerciales a las galerías. \\
- Iniciar un tour de eventos para & Consolidar la reputación de Madrid \\
coleccionistas europeos y americanos, & como una de las grandes capitales \\
combinando una acogedora estancia & culturales a nivel europeo. \\
con charlas y proyectos artísticos, más & - Usar el programa «País Invitado» \\
allá del puro comercialismo de las & como una oportunidad de expandir el \\
ferias. & mercado español y proporcionar a un \\
Encontrar formas de convertirse en una & público amplio una idea más clara del \\
plataforma para mostrar las últimas & arte contemporáneo extranjero. \\
tendencias en el mundo del arte, no sólo & - Estrechar lazos con América Latina. \\
a nivel comercial sino a nivel cultural, & - Internacionalizar la feria y traer \\
ya que el mercado es un seguidor y & importantes coleccionistas mundiales a \\
no un predecesor de los movimientos & la cita de Madrid. \\
culturales más innovadores. & \\
\hline
\end{tabular}


Estas diferencias y similitudes entre las dos marcas se pueden resumir diciendo que, en lo que a marca se refiere, Art Basel ejemplifica la continuidad, mientras que a lo largo de su historia, ARCOmadrid ha necesitado mayores modificaciones. Sin embargo, ambas imágenes de marca han necesitado adaptarse a diseños contemporáneos que reflejaran los cambios en la visión y la misión de las dos organizaciones (desde el ámbito puramente comercial de los 80 hacia el evento cultural en los 90, y luego hacia la más ambiciosa propuesta de convertir la feria en un destino cultural anual).

Así que, "el buen branding debería traducirse en buen diseño, porque, a menudo, es el logo de la organización, el folleto o la web lo que se conoce o se recuerda ${ }^{6}$ y los principales elementos visuales de la marca son: nombre y logo.

Hablando del primero, ambas ferias tienen nombres descriptivos: Art Basel y ARCOmadrid son únicos (Arco exclusivamente es el nombre de una compañía de seguridad en el Reino Unido y una compañía de gasolineras en los Estados Unidos); fáciles de pronunciar; simples y fáciles de recordar; dan una idea de la categoría del producto/servicio al que se refieren (Art Basel directamente menciona el sector cultural); adaptable a distintos idiomas y susceptibles de protección legal.

Refiriéndonos al logo, el de Art Basel refleja los mismos valores desde que se creó la feria y ha alcanzado reconocimiento de marca; es la feria más importante del mundo dedicada al arte contemporáneo internacional y su mayor objetivo es mantener la promesa de calidad en el siglo XXI, expandiendo el mercado a los consumidores asiáticos, liderando la escena cultural e introduciendo herramientas tecnológicas que mejoren la experiencia global del evento. ARCOmadrid, por otro lado, necesitó hacer cambios estratégicos en 2009 para incluir el nombre de Madrid en el logo y simplificarlo (el país invitado ya no aparece). Este cambio de imagen refleja las transformaciones internas que pretenden redefinir la promesa al consumidor: intentando atenuar los efectos negativos de la crisis económica, impulsando una mayor presencia del arte latinoamericano que se había dejado de lado en favor del programa País Invitado y subrayando la necesidad de promocionar Madrid como destino cultural.

Actualmente, ambos logos tienen un estilo neutro, simple, sobrio y elegante, que refleja la personalidad de la marca. Art Basel lidera el camino con una clara estructura de imagen, basada en el nombre de la ciudad (situando el evento) y el número de veces que se ha celebrado (enfatizando continuidad y

6 NADDAFF, Andrea, "Branding by design", Communication World (online), en: http://www.slideshare.net/RbkAsr/branding-by-design (17-junio-2014). 


\section{DE artículos}

El papel del branding...

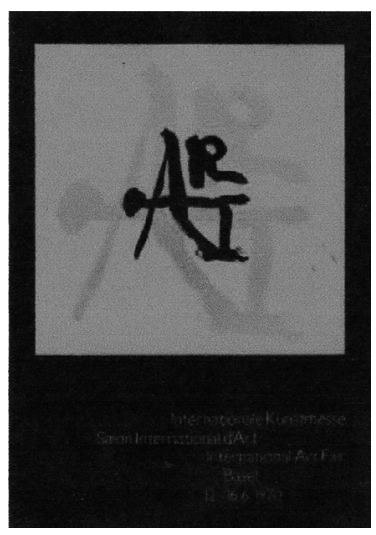

1. Art Basel. Diseño inicial

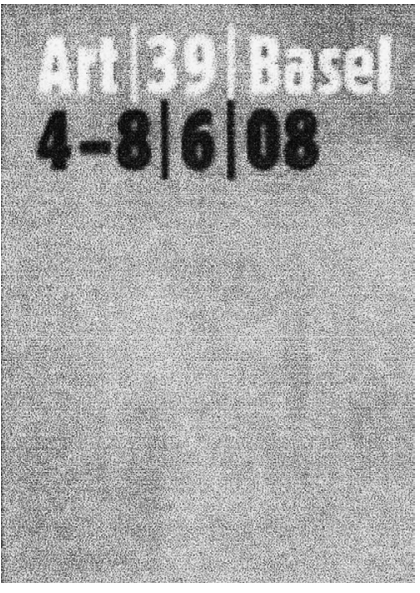

\section{Art|Basel}

Basel| June |13-16 | 2013

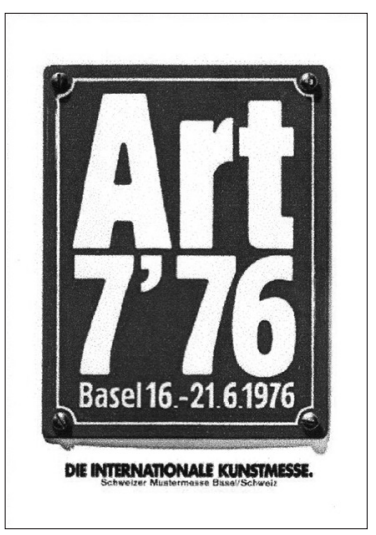

1.1. En 1976 se estableció la tipografía

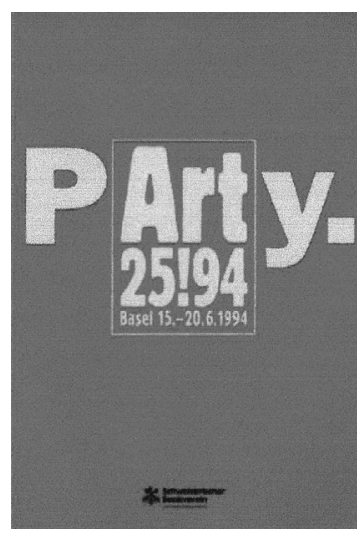

1.2. Primer año de UBS como sponsor

1.3. Desde 2000 la identidad visual está claramente establecida
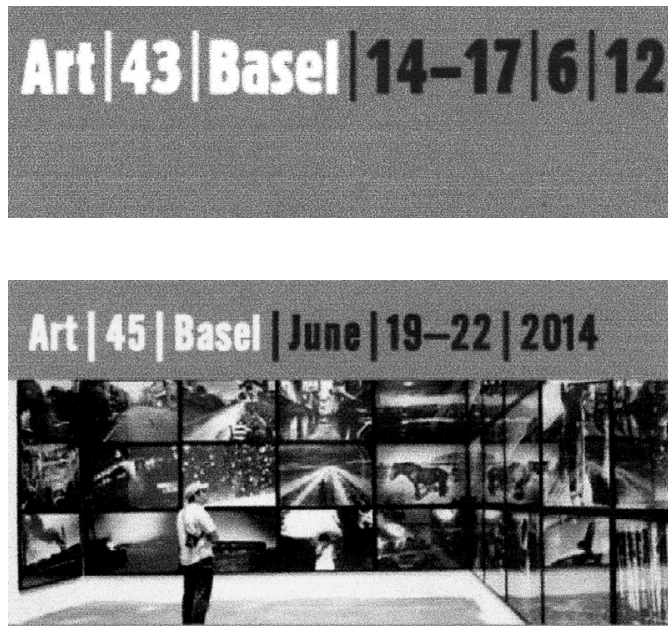

Art| Basel

milaal Beach| Dea|05-08/2014 

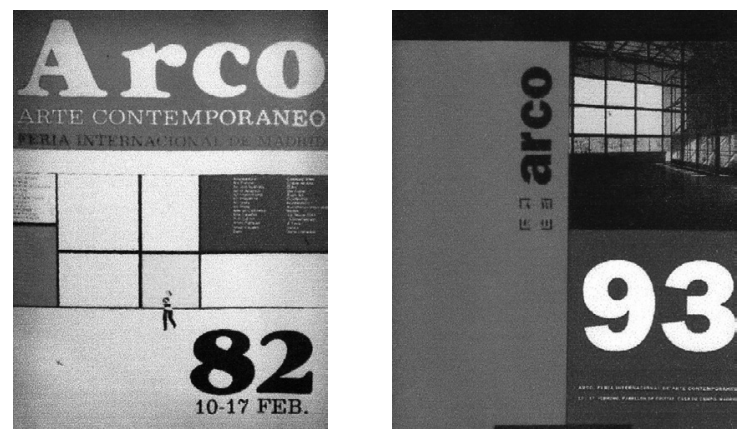

2. ARCOmadrid. Diseño inicial (izq.) y último año (der.) antes de incluir la sección País Invitado
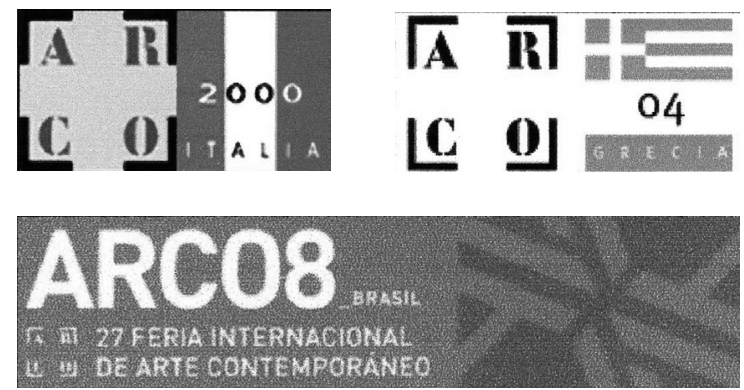

2.1. Entre 1994 y 2009 se enfatizó la presencia del País Invitado

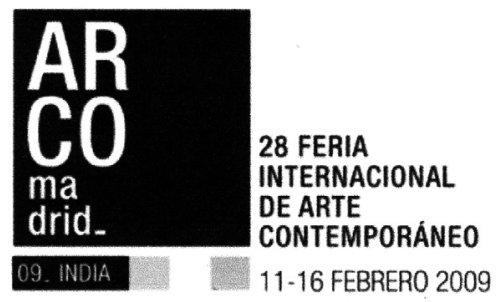

2.2. En 2009 se realiza un cambio de logotipo: pasa a ser un cuadrado negro que mantiene la marca ARCO formando el cuadrado e incorpora Madrid (ciudad de celebración)
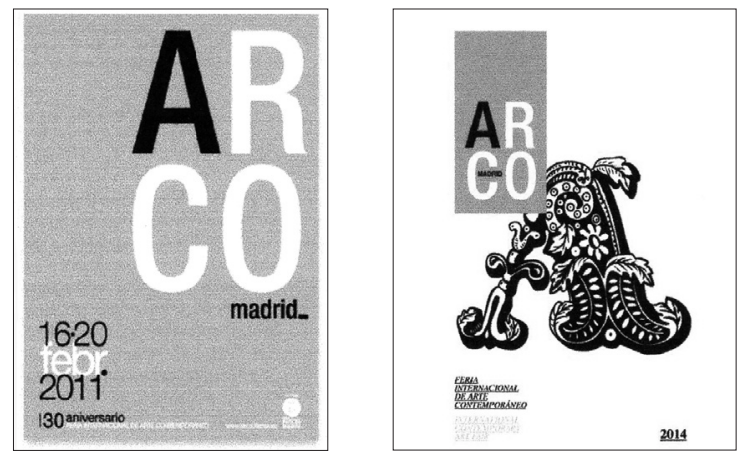

2.3. Carteles de distintas ediciones que juegan con la denominación de la marca ARCO como eje central del visual 
relacionándolo con solidez y éxito); ARCOmadrid ha utilizado el mismo enfoque espacio-temporal, pero sin hacer referencia al número de ediciones de la feria.

A pesar de todo, es importante no perder de vista que ambas organizaciones son ferias de arte (gestionadas en un caso por una compañía privada, $\mathrm{MCH}$ Messe Schweiz, y en el otro por un consorcio público, como es IFEMA ${ }^{7}$ ), que compiten para ser rentables en el negocio del arte y generar resultados económicos positivos cada año, sirviéndose de su valor de marca como un elemento esencial en ese camino hacia el éxito. Por lo tanto, esta es una de las razones por las que actualmente una marca fuerte se ha convertido en una necesidad tan vital en el sector cultural como en el comercial, pero hay otras muchas ventajas asociadas que se analizan en la siguiente sección.

\section{¿Por qué crear una identidad institucional en las ferias de arte?}

La programación artística siempre será fundamental en cada institución, pero conseguir una voz única y un buen posicionamiento puede ayudar de forma definitiva en el camino al éxito Eliza Williams, escritora en Creative Review ${ }^{\circ}$

El negocio del arte mueve billones anualmente en el mundo. Como ejemplo, en el campo del arte contemporáneo, los resultados de las casas de subastas y de las ferias de arte en 2011 fueron lo suficientemente fuertes como para hablar del final de la crisis en un sector que se ve como alternativo a la inversión en bolsa. Según los datos publicados en Artprice, 2012 consolidó la recuperación y en 2013 el arte contemporáneo generó en subasta un $15 \%$ más que el año anterior ${ }^{9}$. Teniendo en cuenta estas cifras, se entiende que "las ferias de arte -como las casas de subastas y galerías antes que ellas- actúan como empresas ${ }^{10}$, intentando consolidar o expandir su posición en el mercado como una plataforma

7 Consorcio formado por: Comunidad de Madrid, Ayuntamiento de Madrid, Cámara de Comercio de Madrid y Caja Madrid.

8 WILLIAMS, Eliza, "Branding the Art World», Creative Review (online), en: http://www.creativereview. co.uk/back-issues/creative-review/2011/october/branding-the-art-world (12-abril-2012).

9 Artprice Trends 2011 indica que las casas de subastas, sobre todo Christie's y Sotheby's, ganaron 2.47 billones de dólares y, de acuerdo con Bloomberg News, Art basel vendió piezas por un total de 1.8 billones de dólares. Artprice Trends 2013, señala un aumento de 140 millones de euros en las ventas de arte contemporáneo mundial aunque comenta el caso español como ejemplo de mercado en recesión con un descenso de 2.787 a 1.047 millones de euros en 2013 y un $70 \%$ de lotes sin adjudicar.

10 GERLIS, Melanie, "Building the brand», The Art Newspaper (online), en: http://www.theartnewspaper.com/articles/Building+the+brand/23992 (21-junio-2014). 
de negocio para las galerías (ventas y contactos). Las ferias compiten para ser más visibles, construyendo una marca que pueda «definir la promesa, describir los medios que la conviertan en una experiencia y [...] asegurar que le llega al público objetivo" ${ }^{11}$.

Así que, una de las principales razones para generar una buena marca es conseguir reconocimiento inmediato en el consumidor objetivo, lo que genera preferencia de marca. ¿Cuántas ferias de arte hay anualmente en el mundo? Incluso, si sólo nos referimos a las de arte contemporáneo, la elección es tan amplia que es esencial destacar, definir aquellos aspectos únicos que consiguen atraer y mantener la lealtad de compradores y visitantes que eligen un evento por encima de los demás. Básicamente, la marca actúa como un identificador (que permite la diferenciación de producto y sirve como indicador de calidad), crea valor percibido que reduce el nivel de riesgo que el consumidor advierte, crea conexiones emocionales y un atajo o simplifica la toma de decisiones del consumidor ${ }^{12}$.

Además, esa posición preeminente tiene un beneficio doble que es decisivo para el sector cultural: atrae consumidores y sponsors.

Consumidores que, en una feria de arte, no son sólo los visitantes sino los coleccionistas, comisarios, asesores, galeristas, directores de museos, críticos, etc. Por lo tanto, en este sector el número de visitantes no es un factor determinante para medir la importancia del evento. Con menos 100.000 visitantes (exactamente 92.000 en la edición de 2014), la posición de liderazgo de Art Basel es indiscutible, mientras que ARCOmadrid ostenta la primera posición en el ranking de visitantes, con ediciones que se han acercado a los 150,000 visitantes ${ }^{13}$. Este éxito muestra cómo la audiencia del siglo XXI genera una gran demanda de productos culturales, no sólo por la parte cultural en sí misma, sino también como una forma de socialización y entretenimiento, para Asker, «tener una relación con una organización y un producto con gran personalidad es satisfactorio y provechoso, porque te

11 VILPONNEN, Zhanna, Branding Artist and Arts Organizations. Sibelius-Akatemia (online), en: http:// ethesis.siba.fi/ethesis/files/nbnfife200906081605.pdf (16-abril-2012).

12 Un caso de marca que no consigue el efecto simplificador sino que genera confusión en el consumidor se da con la marca Armory en Nueva York. The Armory Show es la feria de arte más grande de la ciudad desde 1994 y un evento importante en el calendario internacional. En 2009, la propia feria creó la Armory Art Week, aunando bajo ese nombre todos los eventos culturales de la primera semana de marzo en Nueva York: inauguraciones de museos y 6 ferias de arte, cuyo nombre individual queda diluido (The Armory Show, Independent, Moving Image, Scope, VOLTA NY y ADAA Art Show). Y para aumentar el desconcierto, la última feria mencionada, ADAA Art Show, tiene lugar en el edificio llamado Park Avenue Armory.

13 Posibles explicaciones para este fenómeno serían el que la feria abre el calendario anual, y que todavía mantiene parte de la significación que tuvo en los años 80 cuando supuso un signo de apertura y modernidad después de los años de dictadura. 
Lead Partner

\section{橉 UBS}

Associate Partner

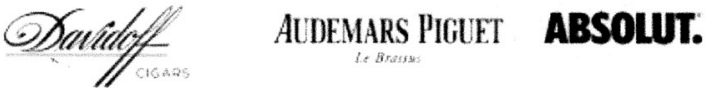

NETJETS

Lounge Host

Kuinart

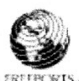

Official Partner

Automotive Hote
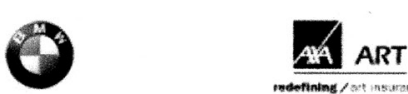

AMES

Hotel

Hotel

MARQUIS

MARQUIS
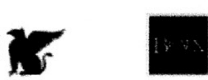

Media Partner

\section{FT}

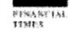

Show Media Partner

\section{Itliami tycralo}

3. Sponsors

de Art Basel

Providers and Supporters

Ar Nexus MIAMIBEACH 


\section{If frame vamalert Nicosit}

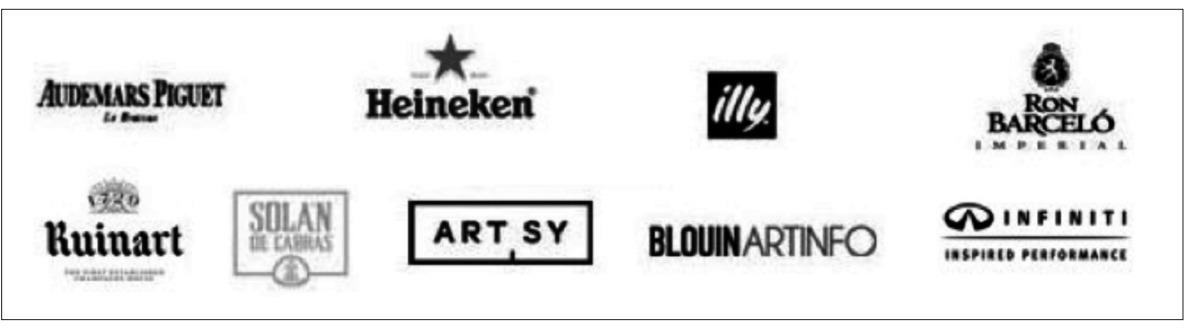

Patrocinadores

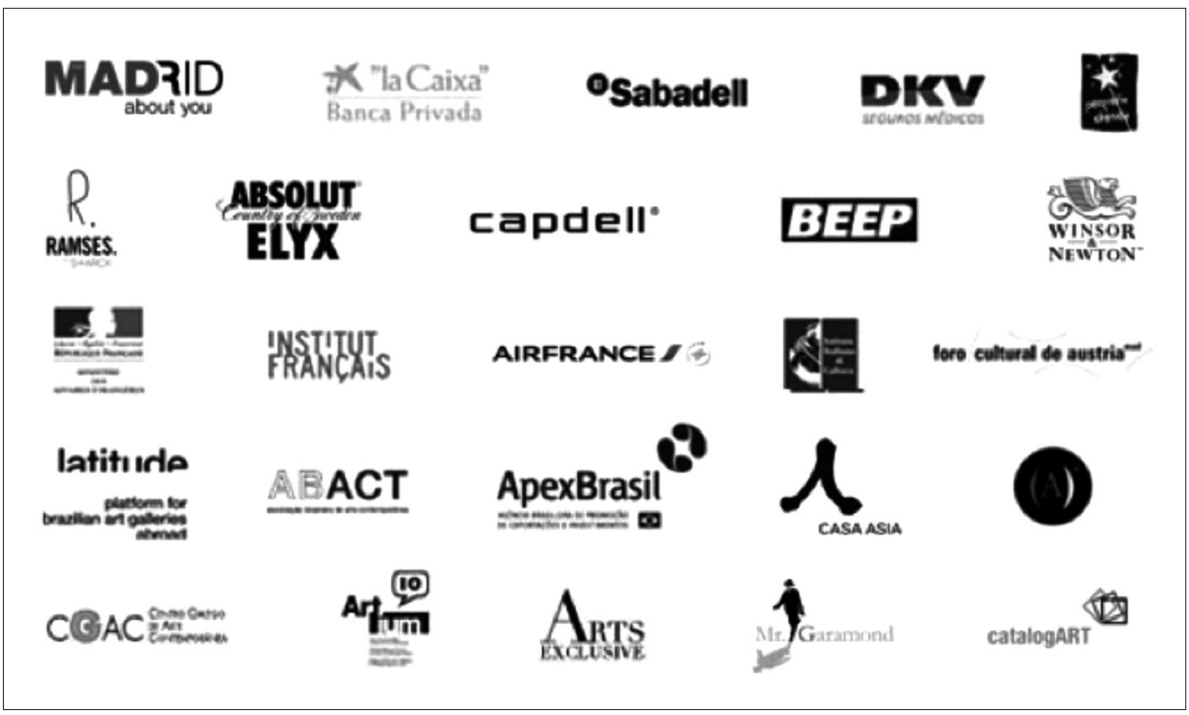

Colaboradores

$\begin{array}{lll}\text { ABC ELeMUNDo ELPAÍs rne } & \begin{array}{l}\text { radio } 3 \\ \text { Oedios }\end{array} \\ \text { Ofiales }\end{array}$

4. Sponsors de ARCOmadrid 
vincula con un grupo de gente que comparte los mismos valores y estilo de vida ${ }^{14}$ (Asker, 1996). Desde el punto de vista del consumidor, la marca «es una forma de expresar sus necesidades sociológicas y psicológicas» ${ }^{15}$ porque cuando un cliente compra una obra de arte, está comprando "un cuadro, por supuesto, pero también un posible cambio en la forma en la que los demás le ven ${ }^{16}$. Esto significa que Madrid y Basilea, durante febrero y junio respectivamente, son los lugares para ver y ser visto en un mercado elitista y exclusivo. Son dos de las citas más esperadas para testar las tendencias del mercado del arte (ARCOmadrid es la primera gran feria del año y Art Basel es en junio, justo después de todas las subastas de arte moderno y contemporáneo y al mismo tiempo que eventos como la Bienal de Venencia o la Documenta de Kassel) o sólo para pasar el día buscando las polémicas obras de arte que recogen las noticias.

Por otro lado, una marca fuerte convierte a la organización de arte en una inversión atractiva para compañías que buscan conectar sus marcas con las connotaciones positivas que la cultura ofrece. Refiriéndose a los casos de estudio, es importante remarcar cómo las ferias de arte no quieren ser consideradas sólo como un acontecimiento comercial sino que intentan ser un evento cultural más amplio, que les permita posicionarse a medio camino entre el negocio y las organizaciones sin ánimo de lucro. En palabras de los directores:

El programa AfterARCO nace con el propósito de llevar los contenidos de la feria a la ciudad de Madrid [...]. Por ello, tras el cierre de puertas de la feria, se abrirá un abanico de posibilidades con instalaciones en espacios públicos, una programación de videoarte por las calles de Madrid, además de sesiones de DJ, conciertos de música pop y electrónica, todo a partir de las 21 horas (Carlos Urroz entrevista para Turismo de Madrid ${ }^{17}$ ).

Dedicamos gran cantidad de tiempo y energía a cosas que no están relacionadas con el mercado, como "Art on Stage», que es un evento teatral gratuito y de entrada libre. Ponemos mucho esfuerzo en "Art Basel Conversations», "Art Lobby», el día de

14 AAKER, David, Building strong brands (online), Londres, Simon \& Schuster, en: http://www.productmanualguide.com/strong/building-strong-brands.html (22-junio-2014).

15 COLMENARES, Óscar, "Posicionamiento y marcas», Gestiopolis (online), en: http://www.gestiopolis. com/marketing/el-concepto-de-marca-en-la-empresa-moderna.htm (20-junio-2014).

16 THOMPSON, Don, The \$12 Million Stuffed Shark, Londres, Aurum, 2008, p. 42.

17 Turismo de Madrid, "ARCOmadrid es una feria en evolución», El Madrid de Carlos Urroz (online), en: http://www.turismomadrid.es/el-madrid-de/11634-carlos-urroz-arcomadrid-es-una-feria-en-evolucion (19-junio-2014). 
visitas profesionales y otros muchos aspectos que no son sólo la parte comercial de la feria (Annette Schnholzer and Marc Spiegler entrevista para Artinfo ${ }^{18}$ ).

Por lo tanto, se necesitan sponsors para poder financiar todas las actividades generadas alrededor de la feria, creando, como contrapartida, un ambiente perfecto para establecer o mantener contactos directos y significativos con clientes y potenciales clientes:

- Art Basel: UBS ha sido el principal sponsor desde 1994 «reflejando el deseo de la firma de desarrollar asociaciones globales, duraderas e innovadoras que contribuyan a conseguir la diferenciación de UBS como marca» ${ }^{19}$, lo que se aprecia en toda la feria, incluyendo el catálogo y la página web.

- ARCOmadrid: recibe dinero público y privado. La inversión pública supone un intento de convertir la cultura en algo más accesible (descuentos para estudiantes, entrada reducida el último día de la feria...) y busca mejorar el perfil de Madrid internacionalmente. En cuanto a sponsors privados, la Fundación Banco Santander, por ejemplo, patrocina los Encuentros Profesionales de la feria como parte de su programa de patrocinio cultural.

Finalmente, ferias de arte con una marca clara y potente son sinónimo de calidad y eventos imprescindibles cuyos nombres añaden procedencia y valor a la obra de arte, lo que significa excelencia a nivel de producto y de experiencia. "Acogen a los galeristas más importantes, que quieren participar porque las mejores ferias siempre atraen a los coleccionistas más relevantes. Éstos a su vez visitan la feria porque los galeristas más potentes están en ellas. Esto es lo que los economistas Ilaman paradoja de retroalimentación que lleva a un oligopolio que se auto perpetúa entre las ferias más importantes ${ }^{20}$ con un doble efecto: las mejores galerías están interesadas en formar parte de la feria, porque por ejemplo, «el respaldo de la marca Basel ofrece seguridad a los galeristas internacionales, que actualmente tienen que elegir entre una gran cantidad de ferias cada año»" y la feria puede anunciar orgullosamente la participación de esas afamadas ga-

18 Artinfo, "Annette Schnholzer and Marc Spiegler», International Edition (online), en: http://uk.blouinartinfo. com/news/story/270670/annette-schnholzer-and-marc-spiegler (22-junio-2014).

19 UBS news, en: http://www.ubs.com/global/en/about_ubs/about_us/news/news.html/en/2003/07/17/ news_07-17-2003.html (22-junio-2014).

20 Esto muestra como las mejores marcas ofrecen, además, una fuerte defensa contra nuevos competidores. THOMPSON, Don, The $\$ 12$ Million Stuffed Shark..., p. 56.

21 GERLIS, Melanie, "Building the brand», The Art Newspaper (online), en: http://www.theartnewspaper.com/articles/Building+the+brand/23992 (21-junio-2014). 
lerías que necesitan presentar un programa de gran calidad para ser aceptadas. La promesa que la feria le hace al consumidor es que sólo se ofrecen piezas de la más alta calidad, lo que significa que los coleccionistas esperan impacientes a ver los stands y saben que pueden comprar con total confianza porque las obras han pasado por un largo y estricto proceso de selección. El nombre de la feria se convierte en una garantía ${ }^{22}$; todo depende de que la organización sea capaz de producir una experiencia global inimitable que destaque por la fuerza del producto que ofrece.

Un producto, que debido a su propia naturaleza obliga al sector cultural a vender experiencias además de productos físicos, una obra de arte no tiene sólo un valor económico sino un valor cultural que Throsby explica como la suma de el valor estético (belleza y armonía), el valor espiritual (pertenencia a un colectivo), el valor social (sentido de identidad), el valor histórico (continuidad con el pasado), el valor simbólico (como transportador de significado), y el valor de autenticidad (de un producto único e inseparable del artista y el consumidor) ${ }^{23}$.

Experiencia y consumidor son las dos palabras clave íntimamente ligadas a el branding y el marketing de las organizaciones de arte.

\section{Cumpliendo la promesa: el papel del branding en el marketing}

La puesta en práctica de la estrategia de marketing es definitiva para el éxito o el fracaso de la marca en el momento de mayor importancia para el consumidor: el momento en el que experimentan la marca a través de anuncios, promociones, compra, uso y servicio de postventa.

Van Gelder ${ }^{24}$

La marca es el vínculo de unión; es la encargada de sintetizar la misión y la visión de una organización, y de colocar esa misión y visión en la posición precisa para que el marketing pueda trasladarlos al consumidor y conseguir "una experiencia

22 Las ferias facilitan la posibilidad de crear y mantener una relación con los galeristas, especialmente con programas como Nuevos Coleccionistas en ARCOmadrid, que ofrece un servicio de consultoría de arte para gente que no es compradora habitual.

23 THROSBY, David, Economics and Culture (online), en: http://books.google.es/books?id=msDdVdklvD $A C \&$ printsec $=$ frontcover\&hl=es\#v=onepage\&q\&f=false $(22$-junio-2014).

24 VAN GELDER, Sicco, Global Brand Strategy, Londres, Kogan Page, 2008, p. 16. 
total de marca» ${ }^{25}$. Sin embargo, es discutible que esto sea tan simple porque: primero, la marca sólo tiene sentido por y para el consumidor -es la idea que el consumidor tiene sobre la organización-y la percepción del consumidor puede verse influida por factores externos fuera del control del marketing; y en segundo lugar, porque es un proceso circular en el que el marketing comunica la marca pero al mismo tiempo la alimenta o la destruye, porque, como explica Michael Eisner, antiguo director de la compañía Walt Disney "una marca es una entidad viva, que se enriquece o debilita con el tiempo, a causa de muchos pequeños gestos".

Pequeños gestos determinantes en todos los empleados de la compañía y cuyo objetivo debe ser cumplir con la promesa de marca en cada ocasión, en cada interacción con el consumidor, creando experiencias positivas de forma consistente y especialmente durante la feria, que es el momento en el que realzar el aura de la marca. Una marca tiene que liderar el plan de marketing de la organización para conseguir una relación duradera con los consumidores, a través de la creación de lealtad a la marca. La marca tiene que definir todos los productos de marketing, los proyectos y las campañas, siempre orientadas a intentar satisfacer en mayor medida las aspiraciones del mercado objetivo.

Y ¿quién es el mercado objetivo en una feria de arte? Considerando todo lo mencionado anteriormente, la conclusión es que los directores de las ferias de arte están intentando ampliar y democratizar la audiencia a base de convertir el evento en algo más accesible y no tan centrado en la parte puramente comercial. Sin embargo, ¿esto es algo real o el verdadero objetivo es crear una experiencia más completa para aquellos que ya acudían a la feria? Después de todo, diversidad no es una palabra muy utilizada en el sector comercial del mundo del arte, excepto cuando se refiere a nuevos mercados (países emergentes como China, Brasil o India). Si se trata sólo de número de visitantes ¿por qué Art Basel con menos visitantes tiene más de 1.000 galerías de todo el mundo compitiendo por participar y no prefieren tener un stand en ARCOmadrid que acoge más de 100.000 personas al año? Es cierto, que desde el punto de vista de la organización la venta de entradas puede ser un éxito pero ya hemos señalado que este tipo de eventos se centra en la calidad sobre la cantidad.

Un ejemplo práctico de cómo servirse de la marca en la estrategia de marketing es este email que Christie's Education Londres envía a sus estudiantes:

25 KYLANDER, Nathalie y STONE, Christopher, "The role of branding the non-profit sector", Stanford Social Innovation Review (online), en: http://bc2.berkeley.edu/wp-content/uploads/2012/07/the-role-ofbrand-in-the-nonprofit-sector.pdf (22-junio-2014). 
«Art Basel: No sólo ir, sino pertenecer»

\section{VIAJE DE ESTUDIO A ART BASEL}

Únete a nosotros en este exclusivo viaje de estudio de 6 días $/ 5$ noches a la feria suiza Art Basel. Visitando Zurich y Basilea, este excepcional viaje coincide con la feria Art Basel, un evento imprescindible para los amantes del arte contemporáneo internacional.

Cuidado alojamiento en hoteles con encanto, transporte en Suiza y la mayoría de las comidas incluidas. Plazas limitadas a 15 para asegurar una experiencia privada y con acceso a los espacios exclusivos.

Momentos destacados del viaje:

- Entrada a la inauguración de Art Basel.

-Visitas especiales a la feria: acceso a la jornada "sólo con invitación».

- Visita a la Fundación Beyeler.

- Visitas privadas a museos y galerías en Basilea y Zurich.

- Acceso a colecciones privadas no abiertas al público.

Aquí se puede ver la asociación estratégica de estas dos exclusivas marcas, Christie's y Art Basel, colaborando para ofrecer un viaje de estudio VIP a la feria y a otros lugares de interés (nombrando también importantes marcas como la Fundación Beyeler). Este es un mail directo, enviado utilizando la base de datos de Christie's a un público de clase media-alta, con educación superior, interesados en el mundo del arte y con una buena red de contactos; una invitación limitada a 15 asistentes para asegurar calidad, poniendo precio a la experiencia única de asistir a la inauguración y al día «sólo con invitación».

Este es el tipo de herramienta de marketing que se espera para estos eventos. Se usan formas de comunicación a medida para alcanzar el núcleo del público objetivo de la feria de arte: compradores o potenciales compradores son contactados por mails directos, invitaciones personales o llamadas telefónicas desde la organización o las propias galerías, mientras que un abanico más amplio de medios se utilizan para atraer a los visitantes a los días de público general, esos días en los que la feria se convierte en un museo efímero: redes sociales, anuncios en periódicos nacionales y revistas especializadas en arte, newsletters, etc. Es interesante señalar cómo los materiales de divulgación de Art Basel señalan que la feria está abierta cuatro días, aunque la inauguración 
y los días sólo con invitación empiezan dos días antes. Sólo se hace referencia a los días públicos porque la exclusividad de todos los eventos anteriores hace innecesaria la publicidad. En cambio, ARCOmadrid sí que dura cinco días, incluyendo los dos primeros días que son sólo para visitas profesionales ${ }^{26}$.

Así que branding y marketing juegan su parte en crear y comunicar experiencias significativas en el interminable proceso de mejorar la satisfacción del consumidor y permitir a las organizaciones de arte el alcanzar sus metas. Como parte del proceso, una evaluación de marca específicamente diseñada después de cada evento ayudará a incrementar la eficacia del marketing.

Entonces, ¿cuál es el papel que la marca juega en las organizaciones de arte? Scheff resume la respuesta a esta pregunta cuando afirma, "una marca es al marketing lo que la misión es a la organización. La marca dirige toda la función del marketing ${ }^{27}$. Para las ferias de arte, esto significa que deben perseguir no sólo la excelencia del producto sino de la experiencia global al tiempo que mantienen la exclusividad, utilizando en su beneficio el factor de escasez del producto y haciendo un uso estratégico del factor de posicionamiento. Estos factores se deben aplicar no sólo a la experiencia de la feria en sí misma, sino a cualquier punto de contacto con el que la marca se asocie, a lo largo de todo el proceso.

26 La estrategia de venta de entradas de las ferias de arte es solo relevante para el público general. Ambas ferias utilizan diferentes estrategias de precios: las entradas generales de Art Basel cuestan $45 \mathrm{CHF}(37 €)$, salvo las de última hora de la tarde que son $25 \mathrm{CHF}$ ( $20 €$ de 17.00 a $19.00 \mathrm{~h}$ ). Mientras que ARCOmadrid cuesta $40 €$ el viernes y sábado y $30 €$ el domingo, sin contar descuentos para estudiantes. Así que, incluso aunque estos eventos están intentando ampliar su público potencial, todavía hay una clara barrera de exclusividad entre conocedores con invitación (coleccionistas y profesionales) y público general.

27 SCHEFF, Joanne, Arts Marketing Insights: The Dynamics of Building and Retaining Performing Arts Audiences. San Francisco, Jossey-Bass, 2007, p. 193. 